\title{
Silencing of ANXA3 expression by RNA interference inhibits the proliferation and invasion of breast cancer cells
}

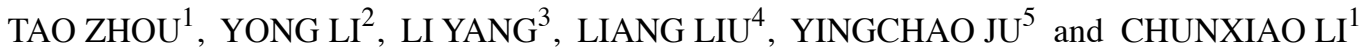 \\ ${ }^{1}$ Breast Disease Center, and Departments of ${ }^{2}$ General Surgery and ${ }^{3} \mathrm{CT}$, ${ }^{4}$ Tumor Institute, and \\ ${ }^{5}$ Animal Experimental Center, The Fourth Hospital of Hebei Medical University, \\ Shijiazhuang, Hebei 050011, P.R. China
}

Received May 28, 2016; Accepted July 8, 2016

DOI: $10.3892 / o r .2016 .5251$

\begin{abstract}
The present study aimed to explore the expression of Annexin A3 (ANXA3) in breast cancer cells and the mechanisms involved in the regulatory effects of ANXA3 on proliferation, invasion and migration of breast cancer cells. Fluorescence quantitative reverse transcription-polymerase chain reaction (qRT-PCR) and western blotting were used to measure the expression of ANXA3 mRNA and protein in two breast cancer cell lines (MDA-MB-231 and MCF-7). Three ANXA3 silencing shRNA plasmids (ANXA3-sh1-3) and one negative control plasmid were constructed, and the Lipofectamine transfection method was used for transfecting human breast cancer cell line MDA-MB-231. Flow cytometry was used to measure the transfection efficiency. The expression of ANXA3 protein was measured by western blotting. Cell cycle distribution and apoptosis were assessed by flow cytometry. Migration and invasion of the transfected cells were evaluated using wound healing and Transwell assays, respectively. The expression levels of ANXA3 mRNA and protein were significantly higher in the MDA-MB-231 cells than levels in the MCF-7 cells. Western blotting showed that the ANXA3 protein level was significantly lower in the MDA-MB-231-Sh cells than that in the MDA-MB-231 and MDA-MB-231-NC cells. In addition, the percentage of $\mathrm{G}_{0 / 1}$ cells and the apoptosis rate were significantly higher, while the cell proliferation rate was significantly lower, in the MDA-MB-231-Sh cells when compared with the MDA-MB-231-NC and MDA-MB-231 cells. The cell migration and invasion abilities were also lower in the MDA-MB-231-Sh cells than these abilities in the MDA-MB-231-NC and MDA-MB-231 cells. The present study investigated the relationships between ANXA3 and prolifera-
\end{abstract}

Correspondence to: Dr Yong Li, Department of General Surgery, The Fourth Hospital of Hebei Medical University, Shijiazhuang, Hebei 050011, P.R. China

E-mail: li_yong_hbth@126.com

Key words: Annexin A3, breast cancer, migration, proliferation, RNA interference tion, apoptosis, migration and invasion of breast cancer cells to elucidate the mechanisms involved in the development, progression, invasion and metastasis of breast cancer.

\section{Introduction}

Breast cancer is one of the most common cancers among women, and its incidence is increasing every year. Breast cancer accounts for $25-30 \%$ of all the cases of malignancy among women in Europe and the USA. In China, breast cancer is now the first leading malignancy among women. The invasion, metastasis and recurrence of breast cancer affect the survival and the quality of life of patients. Therefore, it is important to investigate the mechanisms involved in the development, progression, invasion, metastasis and recurrence of breast cancer for treating this disease and improving the survival of patients. The Annexin A3 (ANXA3) family is a family of $\mathrm{Ca}^{2+}$-dependent phospholipid- and membrane-binding proteins $(1,2)$. ANXA3 plays a role in membrane transport and other calmodulin-dependent activities on the membrane surface. It is involved in regulating inflammatory responses, cell differentiation, and interactions of cytoskeletal proteins. The expression of ANXA3 is associated with multiple human diseases. For instance, Annexin can mediate cell signaling pathways, cell movement, tumor invasion and metastasis, cell apoptosis, and drug resistance in the development and progression of tumors (3-6). As a member of the Annexin family, ANXA3 plays an important role in tumorigenesis, cell proliferation, apoptosis, invasion, metastasis and drug resistance $(7,8)$. However, the effects and roles of ANXA3 in breast cancer are still unclear. The present study investigated the relationships between ANXA3 and proliferation, apoptosis, migration and invasion of breast cancer cells to elucidate the mechanisms involved in the development, progression, invasion and metastasis of breast cancer.

\section{Materials and methods}

Cell lines. MDA-MB-231 and MCF-7 cell lines were passaged and cultured in RPMI-1640 medium containing 10\% fetal bovine serum (FBS) and $100 \mathrm{U} / 1$ of penicillin and streptomycin. The cells were cultured in an incubator at $37^{\circ} \mathrm{C}$ in $5 \%$ $\mathrm{CO}_{2}$. 
Reagents and equipment. Lipofectamine 2000 was purchased from Invitrogen (Carlsbad,CA, USA). Polyvinylidene difluoride (PVDF) membrane and agarose were obtained from Bio-Rad (Mississauga, ON, Canada). RPMI-1640 culture medium and bovine serum albumin were procured from Gibco-BRL (Life Technologies, Paisley, Auckland). Primers were obtained from Sangon (Shanghai, China). The High Purity Plasmid Miniprep kit was purchased from Tiangen Biochemical Technology Co., Ltd. (Beijing, China). RNAiso total RNA extraction reagent, HiScript II First Strand cDNA Synthesis kit, and qPCR SYBR-Green Master Mix were procured from Vazyme Biotech (Nanjing, China). The Annexin V-PE/7-AAD kit and Matrigel matrix basement membrane were purchased from BD (San Diego, CA, USA), and Transwell 3422 was obtained from Corning (Tewksbury, MA, USA). The equipment used in the present study included an Epics-XL flow cytometer (Beckman Coulter, Miami, FL, USA), polymerase chain reaction (PCR) instrument (Eppendorf, Hamburg, Germany), and an Mx3000P fluorescence quantitative PCR instrument (Agilent, Santa Clara, CA, USA).

Assessing the expression of ANXA3 mRNA using fluorescence quantitative RT-PCR. MDA-MB-231 and MCF-7 cells that grew well and were in the logarithmic phase were collected and washed with cold phosphate-buffered saline (PBS) twice. Then, $1 \mathrm{ml}$ of RNA isolater was added to extract total RNA using the one-step method. cDNA was obtained by reverse transcription, which was used as the template for PCR. Human glyceraldehyde-3-phosphate dehydrogenase (GAPDH) was used as the internal reference for standardization. SYBRGreen I was used as the fluorescent dye. Real-time PCR was performed according to the standard protocol. The experiments for each sample were repeated thrice. The forward primer for ANXA3 was, 5'-TCCGAAACATCTGGTGAC-3' and the reverse primer was, 3'-TCAAGTTCTTCGTAAT ACCGAT-5'; the forward primer for GAPDH was, 3'-CACT ACCGTACCTGACACCA-5', and the reverse primer was 3'-ATGTCGTTGTCCCACCACCT-5'. The $2^{-\Delta \mathrm{Ct}}$ method was used to calculate the relative expression level of ANXA3 $\operatorname{mRNA}\left(\Delta \mathrm{Ct}=\mathrm{CT}_{\mathrm{ANXA3}}-\mathrm{CT}_{\mathrm{GAPDH}}\right)$.

Assessing the expression of ANXA3 protein using western blotting. MDA-MB-231 and MCF-7 cells that grew well and were in the logarithmic phase were collected and washed with cold PBS twice. The cell lysis solution was added to collect total protein, and the bicinchoninic acid assay was used to evaluate the protein concentration. Sodium dodecyl sulfate-polyacrylamide gel electrophoresis $(10 \%, 50 \mu \mathrm{g} / \mathrm{well})$ was used for separation, and the protein was transferred onto the PVDF membrane, which was then incubated overnight at $4^{\circ} \mathrm{C}$ with $5 \%$ skimmed milk. Then, 1:500 diluted mouse anti-human ANXA3 monoclonal antibody or 1:4,000 diluted rabbit anti-human $\beta$-actin monoclonal antibody was added. After incubating at room temperature for $1 \mathrm{~h}$, the membrane was washed thrice. Next, 1:20,000 diluted fluorochrome-labeled secondary antibody was added and incubated at room temperature for $2 \mathrm{~h}$. The membranes were imaged using the infrared imaging system. $\beta$-actin was used as the internal reference to analyze the relative expression level of the ANXA3 protein. The experiment was repeated thrice.
Table I. shRNA targets for the ANXA3 gene.

\begin{tabular}{ll} 
No. & \multicolumn{1}{c}{ Target sequence } \\
\hline 1 & GGACAAGCAGGCAAATGAAGG \\
2 & GCATTATGGCTATTCCCTATA \\
3 & GAGATGACATTAGTTCCGAAA \\
\hline
\end{tabular}

ANXA3, Annexin A3.

Construction of the RNA interference lentiviral vector. pGMIV-SC5 was used for constructing the RNA interference lentiviral vector. Restrictive endonucleases BamHI (GGATCC) and EcoRI (GAATTC) were used to linearize the vectors, and then target RNAi sequences were connected to construct the lentiviral vector with target RNAi sequences.

Selection of the shRNA target: the RNAi sequences for ANXA3 gene were designed using the public network. Multiple targeting RNAi sequences were designed in the present study, and then the sequences with the best kinetic parameters were selected, according to the experiences and the evaluation by the software, for the consequent experiments. The shRNA target sequences are listed in Table I.

Design of the shRNA primer: oligomeric single-stranded DNA for the shRNA was designed and synthesized according to the gene sequences. The oligo sequences are shown in Table II.

Construction of the reconstructed RNAi lentiviral plasmid. The oligomeric single-stranded DNA was annealed to obtain a double-stranded shRNA oligo which was inserted into the shRNA lentiviral vector to construct the reconstructed shRNA lentiviral plasmid, and then competent DH5 $\alpha$ cells were transfected. Several single colonies were selected and cultured in the flask. The positive clones were identified by sequencing. The plasmids were extracted after the sequencing confirmed that the plasmids were successfully constructed. After the concentration was measured, the plasmids were stored at $-20^{\circ} \mathrm{C}$ until use.

Transfection of the MDA-MB-231 cells with small hairpin RNA-containing plasmids. The MDA-MB-231 cells were transfected with small hairpin RNA (shRNA)-containing plasmids to silence the $A N X A 3$ gene. The reagents used were as follows. i) Reagent 1: $6 \mu$ l of Lipofectamine 2000 was mixed with $244 \mu \mathrm{l}$ of RPMI-1640 culture medium (FBS- and antibiotic-free), and then placed at room temperature for $5 \mathrm{~min}$; ii) reagent 2: $10 \mu \mathrm{l}$ of shRNA plasmid (containing $4 \mu \mathrm{g}$ plasmid; ANXA3-sh1-3 and negative control plasmids) was mixed with $240 \mu \mathrm{l}$ of RPMI-1640 culture medium (FBS- and antibiotic-free) and then placed at room temperature for $5 \mathrm{~min}$.

The procedures for the cell transfection were as follows. MDA-MB-231 cells in the logarithmic phase were collected and cultured in 6-well plate at the density of $4 \times 10^{5} /$ well. After the cells reached $85-90 \%$ confluence, transfection was performed. The cells were washed with RPMI-1640 medium (FBS- and antibiotic-free) twice, and $2 \mathrm{ml}$ of FBS- and antibiotic-free RPMI-1640 culture medium was added into each 
Table II. Sequences of the oligo DNA for shRNA.

Oligo

Sequence of the oligomeric single-stranded DNA (5' to 3')

5753H_ANXA3-sh1-T (BamHI)

5753H_ANXA3-sh1-B (EcoRI)

5754H_ANXA3-sh2-T (BamHI)

5754H_ANXA3-sh2-B (EcoRI)

5755H_ANXA3-sh3-T (BamHI)

5755H_ANXA3-sh3-B (EcoRI)
GATCCGGACAAGCAGGCAAATGAAGGTTCAAGAGACCTTCATTTGCCTGCTTGTCCTTTTTTG AATTCAAAAAAGGACAAGCAGGCAAATGAAGGTCTCTTGAACCTTCATTTGCCTGCTTGTCCG GATCCGCATTATGGCTATTCCCTATATTCAAGAGATATAGGGAATAGCCATAATGCTTTTTTG AATTCAAAAAAGCATTATGGCTATTCCCTATATCTCTTGAATATAGGGAATAGCCATAATGCG GATCCGAGATGACATTAGTTCCGAAACTCGAGTTTCGGAACTAATGTCATCTCTTTTTT AATTAAAAAAGAGATGACATTAGTTCCGAAACTCGAGTTTCGGAACTAATGTCATCTCG well. The reagents 1 and 2 were mixed gently, placed at room temperature for $20 \mathrm{~min}$, and then added into the wells. The medium was changed to complete culture medium $6 \mathrm{~h}$ later, and a fluorescence microscope was used to observe the cells at $48 \mathrm{~h}$ after the transfection. Untreated cells (blank control group) and cells treated with only Lipofectamine (liposome group) were used as the controls.

Evaluating transfection efficiency using flow cytometry. The cells were collected at $48 \mathrm{~h}$ after the transfection and washed with PBS once. Then, the green fluorescence in the cells was measured using flow cytometry. The percentage of cells expressing green fluorescence (in all the cells observed) was calculated as the transfection efficiency.

Evaluating the expression of ANXA3 mRNA using quantitative reverse transcription- $P C R$. The cells were collected at $48 \mathrm{~h}$ after the transfection, washed with PBS twice, and then treated (as described in the section 'Measuring the expression of ANXA3 mRNA using fluorescence quantitative RT-PCR'). The $2^{-\Delta \mathrm{Ct}}$ method was used to calculate the relative expression level of ANXA3 mRNA $\left(\Delta \mathrm{Ct}=\mathrm{CT}_{\mathrm{ANXA} 3}-\mathrm{CT}_{\mathrm{GAPDH}}\right)$, which was used to assess the silencing effects of the plasmids on the $A N X A 3$ gene. The plasmid with the best interference effects was selected. The experiments were repeated thrice.

Identifying stably transfected cells with puromycin. The experiments showed that the ANXA3 silencing effects of the plasmid ANXA3-sh2 were the best. Thus, this plasmid was chosen for the consequent siRNA experiments. After the MDA-MB-231 cells were transfected with the ANXA3-sh2 plasmid and the negative control plasmid for $48 \mathrm{~h}$, the cells were 1:10 diluted with RPMI-1640 medium and cultured in a 6 -well plate at the density of $5 \times 10^{4} /$ well. Then, $600 \mathrm{ng} / \mathrm{ml}$ of puromycin was added into each well. The non-transfected MDA-MB-231 cells were used as the blank control. After the cells were cultured for 14 days, the surviving cells were considered as stably transfected cells. The cells transfected with ANXA3-sh2 were named as MDA-MB-231-Sh, while the cells transfected with the negative control plasmid were named as MDA-MB-231-NC. Both the MDA-MB-231-Sh and MDA-MB-231-NC cells were cultured in complete culture medium containing $300 \mathrm{ng} / \mathrm{ml}$ of puromycin.

Assessing the expression of ANXA3 protein using western blotting in the transfected cells. The MDA-MB-231,
MDA-MB-231-NC and MDA-MB-231-Sh cells that grew well and were in the logarithmic phase were collected, washed with cold PBS twice, and then treated (as described in the section 'Measuring the expression of ANXA3 protein using western blotting') for western blotting.

Assessing cell cycle distribution using flow cytometry. The MDA-MB-231, MDA-MB-231-NC and MDA-MB-231-Sh cells that grew well and were in the logarithmic phase were collected, washed with cold PBS twice, and fixed with $70 \%$ ethanol at $4^{\circ} \mathrm{C}$ for $24 \mathrm{~h}$. Then, the cells were again washed with PBS twice, and the cell density was adjusted to $1 \times 10^{7} / \mathrm{ml}$. Next, $100 \mu \mathrm{l}$ of cell suspension was collected, and $1 \mathrm{ml}$ of propidium iodide was added. After incubating at $4^{\circ} \mathrm{C}$ for $30 \mathrm{~min}$, flow cytometry was performed and MultiCycle AV software (Beckman Coulter) was used to analyze the cell cycle.

Assessing cell apoptosis using flow cytometry. The MDA-MB-231, MDA-MB-231-NC and MDA-MB-231-Sh cells that grew well and were in the logarithmic phase were collected and washed with cold PBS twice. The cell density was adjusted to $1 \times 10^{7} / \mathrm{ml}$. Then, $100 \mu \mathrm{l}$ of cell suspension was collected, washed with cold PBS once, and suspended in $100 \mu \mathrm{l}$ of $1 \mathrm{X}$ binding buffer. Afterwards, $10 \mu \mathrm{l}$ of Annexin V-PE was added, and the mixture was placed on ice for $15 \mathrm{~min}$ in the dark. Next, $380 \mu \mathrm{l}$ of $1 \mathrm{X}$ binding buffer and $10 \mu \mathrm{l}$ of 7-AAD were added, incubated on ice for $15 \mathrm{~min}$ in the dark, washed with cold PBS once, and suspended with $1 \mathrm{ml}$ of PBS. The apoptosis of the cells was measured using flow cytometry. The EXPO32 ADC software (Beckman Coulter) was used to analyze the immunofluorescence data and evaluate the apoptosis rate.

Evaluating cell migration using the wound healing assay. Transverse lines were drawn at the back of the 6-well culture plate evenly with a marking pen, with the distance of $0.5 \mathrm{~cm}$ between each two lines. Five lines were drawn for each well. Then, $\sim 5 \times 10^{5}$ cells were seeded in each well, which reached $100 \%$ confluence after overnight incubation. On the second day, a $10-\mu l$ pipette tip was used to make scratches vertical to the lines drawn by the marking pen (the same pipette tip was used to make scratches for all the wells). The cells were washed thrice with PBS to remove the unattached cells, and then the serum-free culture medium was added. The cells were cultured in an incubator at $37^{\circ} \mathrm{C}$ and $5 \% \mathrm{CO}_{2}$. Images of the cells were captured at 0 and $24 \mathrm{~h}$. 


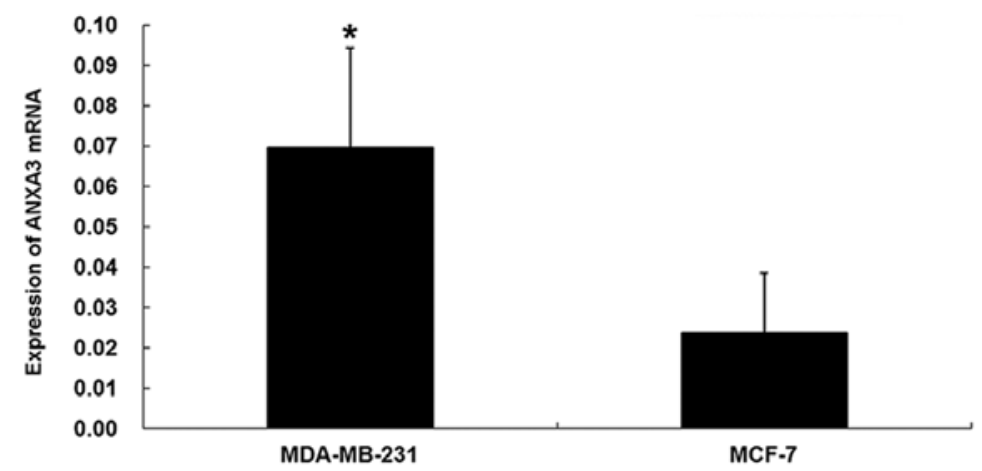

Figure 1. Assessment of the expression of ANXA3 mRNA in the MDA-MB-231 and MCF-7 cells using fluorescence quantitative RT-PCR. Expression of ANXA3 mRNA in the MDA-MB-231 vs. the MCF-7 cells ( $\mathrm{P}<0.01)$.

A

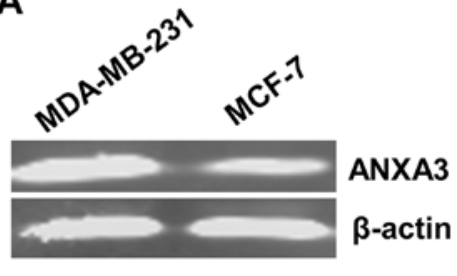

B

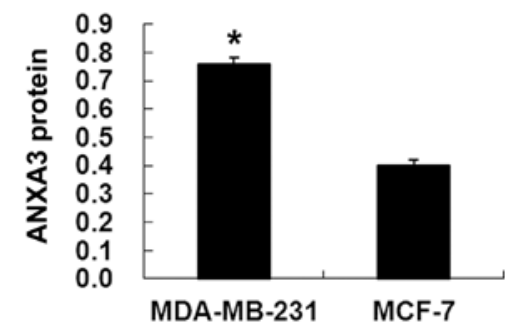

Figure 2. Assessment of the expression of ANXA3 protein in the MDA-MB-231 and MCF-7 cells using western blotting. (A) Western blotting showed that the expression of ANXA3 protein was higher in the MDA-MB-231 cells than that in the MCF-7 cells. (B) Expression of ANXA3 protein in the MDA-MB-231 vs. MCF-7 cells ( $(\mathrm{P}<0.01)$.

Evaluating cell invasion using the Transwell assay. Matrigel and serum-free RPMI-1640 culture medium were mixed (1:8) and used to cover the upper well of the Transwell filter apparatus. Then, $60 \mu \mathrm{l}$ of diluted Matrigel was added into each well and incubated at $37^{\circ} \mathrm{C}$ for $4 \mathrm{~h}$ to allow the Matrigel to turn white. The residual fluid was removed from the culture medium. Next, $50 \mu 1$ of serum-free RPMI-1640 culture medium was added into each well and incubated at $37^{\circ} \mathrm{C}$ for $30 \mathrm{~min}$. The MDA-MB-231, MDA-MB-231-NC and MDA-MB-231-Sh cells that grew well and were in the logarithmic phase were collected, starved in the serum-free medium for $12 \mathrm{~h}$, and then suspended in the serum-free RPMI-1640 medium. Then, $200 \mu \mathrm{l}$ of cell suspension (containing $2 \times 10^{4}$ cells) was added into the upper well, and $600 \mu 1$ of complete culture medium was added into the lower well of the Transwell filter apparatus. The cells were cultured at $37^{\circ} \mathrm{C}$ in $5 \% \mathrm{CO}_{2}$ for $24 \mathrm{~h}$, the upper well was removed, the cells were cleared from the upper well with a cotton swap, crystal violet staining was performed, and the cells that migrated from the upper well to the lower well were counted under a $\times 200$ microscope in five randomly selected visual fields.

\section{Results}

ANXA3 mRNA and protein levels in the MDA-MB-231 and MCF-7 cells. The ANXA3 mRNA and protein levels in the MDA-MB-231 and MCF-7 cells were measured using fluorescence quantitative RT-PCR and western blotting, respectively. The results showed that the relative expression level of the
ANXA3 mRNA was $0.0696 \pm 0.0248$ in the MDA-MB-231 cells, which was significantly higher than the level in the MCF-7 cells $(0.0236 \pm 0.0149 ; \mathrm{P}<0.01 ;$ Fig. 1$)$. The relative expression level of ANXA3 protein was also significantly higher in the MDA-MB-231 cells than that in the MCF-7 cells $(\mathrm{P}<0.01$; Fig. 2). The expression of ANXA3 mRNA and protein was in a parallel trend in the cells.

Construction of the shRNA interference lentiviral vector. The lentiviral vectors were successfully constructed. The sequencing results of the shRNA interference vector are shown in Figs. 3 and 4. The comparison showed that the sequences of the inserted segments were completely identical to the sequences of the designed oligo, suggesting that the lentiviral vectors were successfully constructed.

Silencing of the ANXA3 gene in the MDA-MB-231 cells by the transfection of shRNA plasmid. After the cells were transfected for $48 \mathrm{~h}$, the observation using the fluorescence microscope showed the expression of green fluorescence in the cells transfected with H_ANXA3-sh1, H_ANXA3-sh2, H_ANXA3-sh3, and negative control plasmid. However, the non-transfected cells (blank control) and the MDA-MB-231 cells in the liposome group were found without the expression of green fluorescence (Fig. 5).

Evaluating transfection efficiency using flow cytometry. After the MDA-MB-231 cells were transfected for $48 \mathrm{~h}$, flow cytometry was used to evaluate the transfection efficiency. 
shRNA Sequencing results (shRNA primer shRNA target site)

H_ANXA3 CCTGCAATACGATACAAGGCTGTTAGAGAGATAATTAGAATTAATTTGACTGTAAACACAAAGATATTAGTACAAAATAC $-\operatorname{sh} 1$ GTGACGTAGAAAGTAATAATTTCTTGGGTAGTTTGCAGTTTTAAAATTATGTTTTAAAATGGACTATCATATGCTTACCGTA ACTTGAAAGTATTTCGATTTCTTGGCTTTATATATCTTGTGGAAAGGACGAGGATCO GGACAAGCAGGCAAATGAAGGTTC AAGAGACCTTCATTTGCCTGCTTGTCCTTTTTTGAATTCTAGTTATTAATAGTAATCAATTACGGGGTCATTAGTTCATAGC CCATATATGGAGTTCCGCGTTACATAACTTACGGTAAATGGCCCGCCTGGCTGACCGCCCAACGACCCCCGCCCATTGACG TCAATAATGACGTATGTTCCCATAGTAACGCCAATAGGGACTTTCCATTGACGTCAATGGGTGGAGTATTTACGGTAAACT GCCCACTTGGCAGTACATCAAGTGTATCATATGCCAAGTACGCCCCCTATTGACGTCAATGACGGTAAATGGCCCGCCTGG CATTATGCCCAGTACATGACCTTATGGGACTTTCCTACTTGGCAGTACATCTACGTATTAGTCATCGCTATTACCATGGTGA TGCGGTTTTGGCAGTACATCAATGGGCGTGGATAGCGGTTTGACTCACGGGGATTTCCAAGTCTCCACCCCATTGACGTCA ATGGGAGTTTGTTTTGGCACCAAAATCAACGGGACTTTCCAAAATGTCGTAACAACTCCGCCCCATTGACGCAAATGGGCG GTAGGCGTGTACGGTGGGAGGTCTATATAAGCAGAGCTGGTTTAGTGAACCGTCAGATCCGCTAGCGCTACCGGTCGCCA CCATGGCCCAGTCCAAGCACGGCCTGACCAAGGAGATGACCATGAAGTACCGCATGGAGGGCTGCGTGGACGGCCACAA GTTCGTGATCACCGGCGAGGGCATCGGCTACCCCTTCAAGGGCAAGCAGGCCATCAACCTGTGCGTGTGGAGGGCGGCCC CTTGCCCTTCGCGAGGACATCTTGTCCGCCGCCTTCATGTACGGCAACGCGTGTTCACCGAGTACCCCAGGACATCGTCGA CTACTTCAGAACTCCTGCCCCGCCGGCTACACCTGGGACCGCTTCTTCCTTGTTC

H_ANXA3 CTGGCATTACGATACAGGCTGTTAGAGAGATAATTAGAATTAATTTGACTGTAAACACAAAGATATTAGTACAAAATACGT $-\operatorname{sh} 2$ GACGTAGAAAGTAATAATTTCTTGGGTAGTTTGCAGTTTTAAAATTATGTTTTAAAATGGACTATCATATGCTTACCGTAAC TTGAAAGTATTTCGATTTCTTGGCTTTATATATCTTGTGGAAAGGACGAGGATCCGCATTATGGCTATTCCCTATATTCAAG AGATATAGGGAATAGCCATAATGCTTTTTTGAATTCTAGTTATTAATAGTAATCAATTACGGGGTCATTAGTTCATAGCCCA TATATGGAGTTCCGCGTTACATAACTTACGGTAAATGGCCCGCCTGGCTGACCGCCCAACGACCCCCGCCCATTGACGTCA ATAATGACGTATGTTCCCATAGTAACGCCAATAGGGACTTTCCATTGACGTCAATGGGTGGAGTATTTACGGTAAACTGCC CACTTGGCAGTACATCAAGTGTATCATATGCCAAGTACGCCCCCTATTGACGTCAATGACGGTAAATGGCCCGCCTGGCAT TATGCCCAGTACATGACCTTATGGGACTTTCCTACTTGGCAGTACATCTACGTATTAGTCATCGCTATTACCATGGTGATGC GGTTTTGGCAGTACATCAATGGGCGTGGATAGCGGTTTGACTCACGGGGATTTCCAAGTCTCCACCCCATTGACGTCAATG GGAGTTTGTTTTGGCACCAAAATCAACGGGACTTTCCAAAATGTCGTAACAACTCCGCCCCATTGACGCAAATGGGCGGTA GGCGTGTACGGTGGGAGGTCTATATAAGCAGAGCTGGTTTAGTGAACCGTCAGATCCGCTAGCGCTACCGGTCGCCACCAT GGCCCAGTCCAAGCACGGCCTGACCAAAGGAGATGACCATGAAGTACCGCATGGAGGGCTGCGTGGACGGCCACAAGTTC GTGATCACCGGCGAGGGCATCGGCTACCCCTTCAAGGGCAAGCAGGCCATCAACCTGTGCGTGGTGGAGGGCGGCCCCTT GCCCTTCGCCGAGGACATCTGTCCGCGCCTCATGTACGGCAACGCGTGTTCACGAGTACCCCCAGACATCGTCGACTACTT CAGAACTCTGCCCCGCGCTACACTGGACGCTCTTCTGTCGAGACGGCGCGTGGTGCATCTGCACGCGACATCA

\begin{abstract}
H_ANXA3 CTTGGCAAATACGATACAGGCTGTTAGAGAGATAATTAGAATTAATTTGACTGTAAACACAAAGATATTAGTACAAAATA $-\operatorname{sh} 3$ CGTGACGTAGAAAGTAATAATTTCTTGGGTAGTTTGCAGTTTTAAAATTATGTTTTAAAATGGACTATCATATGCTTACCGT AACTTGAAAGTATTTCGATTTCTTGGCTTTATATATCTTGTGGAAAGGACGAGGATCOGAGATGACATTAGTTCCGAAACT CGAGTTTCGGAACTAATGTCATCTCTTTTTTAATTCTAGTTATTAATAGTAATCAATTACGGGGTCATTAGTTCATAGCCCA TATATGGAGTTCCGCGTTACATAACTTACGGTAAATGGCCCGCCTGGCTGACCGCCCAACGACCCCCGCCCATTGACGTCA ATAATGACGTATGTTCCCATAGTAACGCCAATAGGGACTTTCCATTGACGTCAATGGGTGGAGTATTTACGGTAAACTGCC CACTTGGCAGTACATCAAGTGTATCATATGCCAAGTACGCCCCCTATTGACGTCAATGACGGTAAATGGCCCGCCTGGCAT TATGCCCAGTACATGACCTTATGGGACTTTCCTACTTGGCAGTACATCTACGTATTAGTCATCGCTATTACCATGGTGATGC GGTTTTGGCAGTACATCAATGGGCGTGGATAGCGGTTTGACTCACGGGGATTTCCAAGTCTCCACCCCATTGACGTCAATG GGAGTTTGTTTTGGCACCAAAATCAACGGGACTTTCCAAAATGTCGTAACAACTCCGCCCCATTGACGCAAATGGGCGGTA GGCGTGTACGGTGGGAGGTCTATATAAGCAGAGCTGGTTTAGTGAACCGTCAGATCCGCTAGCGCTACCGGTCGCCACCAT GGCCCAGTCCAAGCACGGCCTGACCAAGGAGATGACCATGAAGTACCGCATGGAGGGCTGCGTGGACGGCCACAAGTTCG TGATCACCGGCGAGGGCATCGGCTACCCCTTCAGGGCAAGCAGGCCATCATCTGTGCGTGTGAGGGCGGCTCCTTGCCCTT CGCGAGGACATCTTGTCCGCGCCTTCATGTACGCACGCGTGTCACGAGTACCCCAGACATCGTCGACTACTTCAGACTCCT GCCCGCGGCTACCCCTGGGACCGCTCCTTCCTGTTCGAGA
\end{abstract}

Figure 3. Sequencing results of the shRNA interference lentiviral vector.

The results showed that the transfection efficiency was $82.97 \pm 0.37,85.25 \pm 1.07,82.33 \pm 0.32$ and $81.41 \pm 0.28 \%$ in the
H_ANXA3-sh1, H_ANXA3-sh2, H_ANXA3-sh3 and negative control groups, respectively. The transfection efficiency 


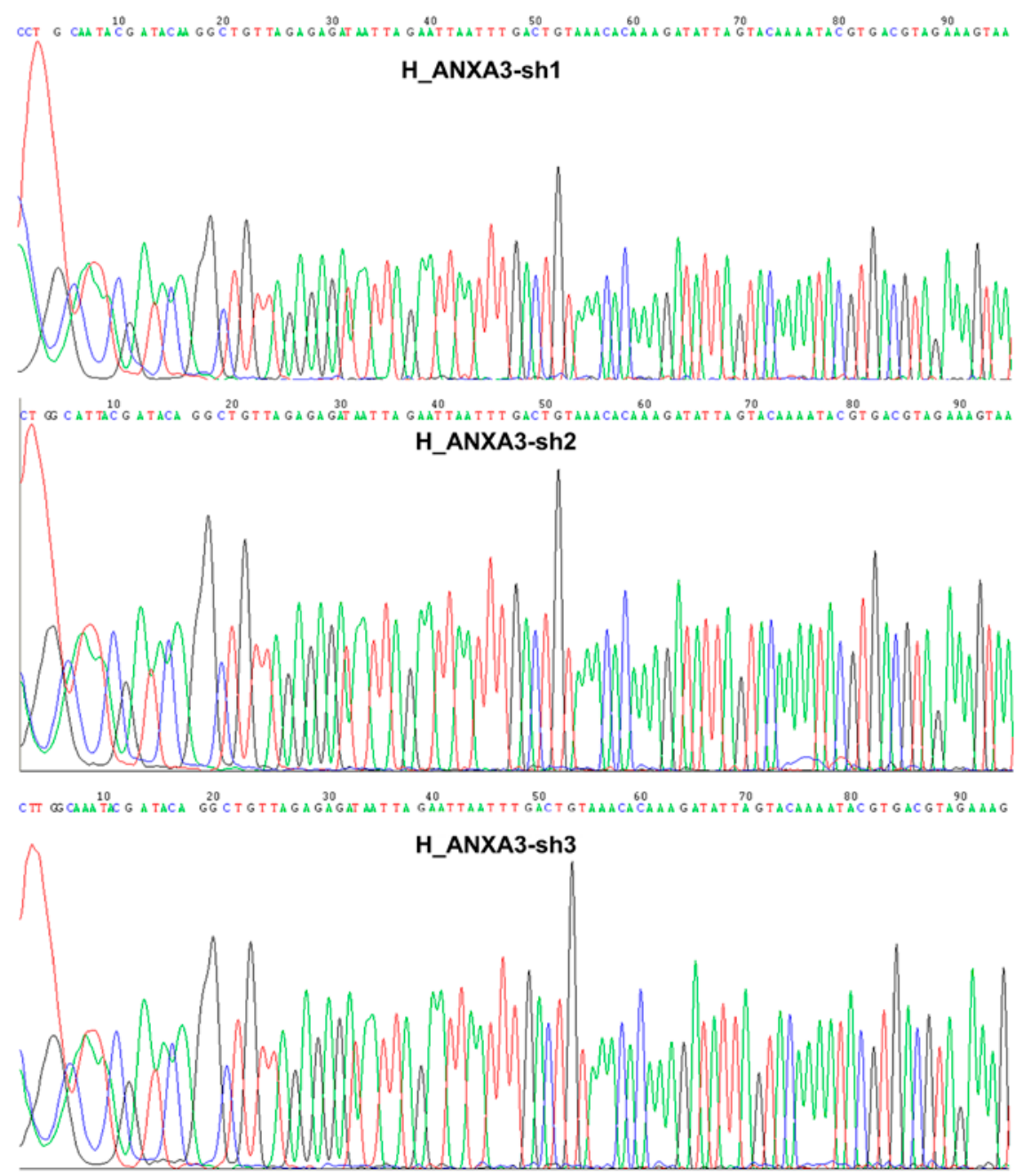

Figure 4. Sequences of the shRNA interference lentiviral vector (part).
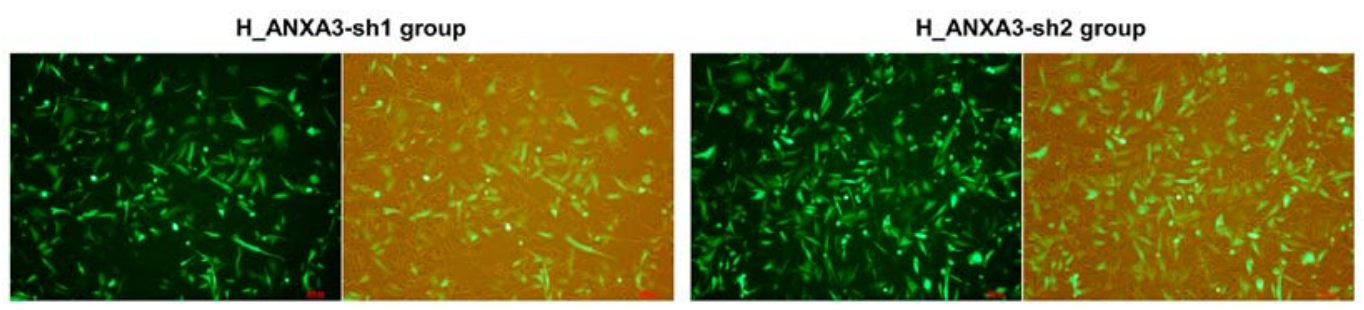

H_ANXA3-sh3 group

Negative control group
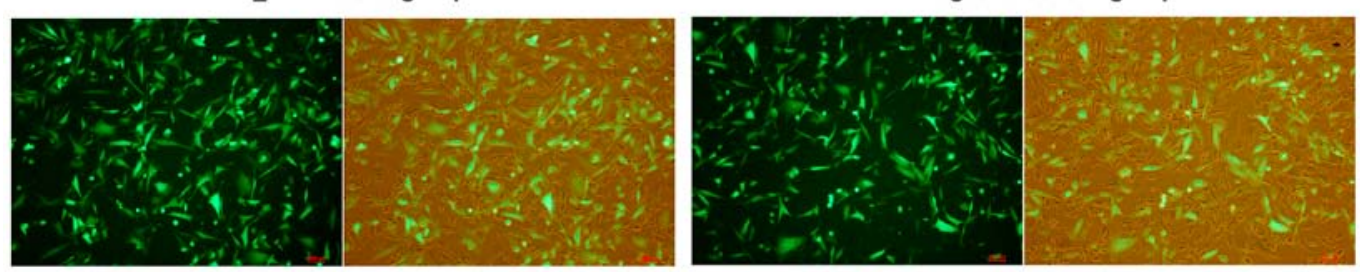

Liposome group

Blank control group
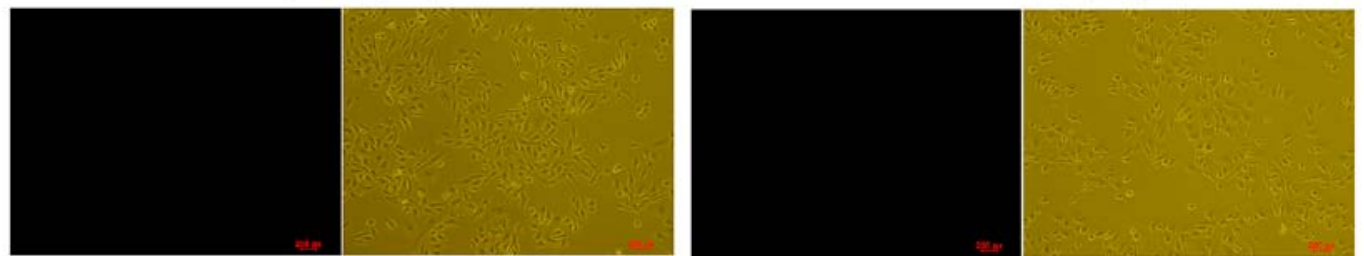

Figure 5. Observation of the expression of green fluorescence using the a fluorescence microscope (magnification, x100). 


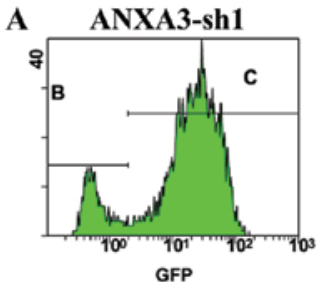

GFP

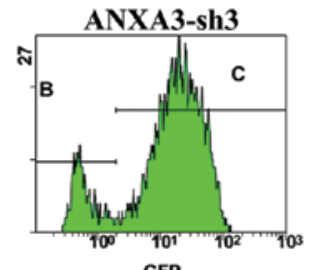

GFP

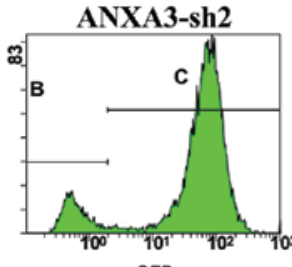

GFP

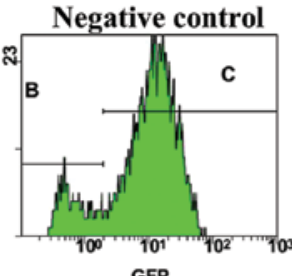

GFP
B

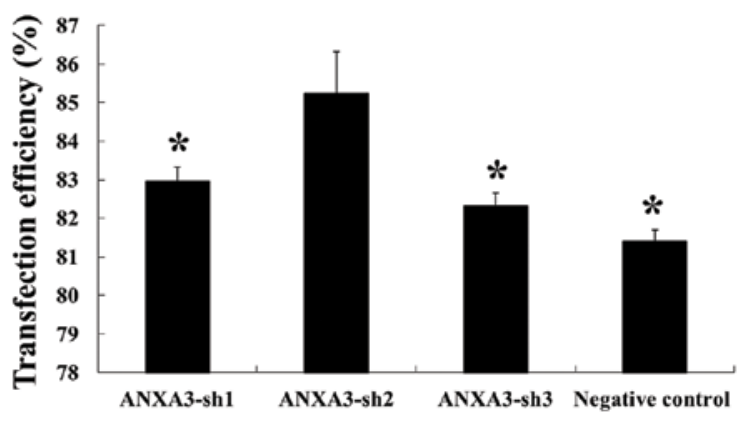

Figure 6. (A) Evaluating the transfection efficiency using flow cytometry. (B) The percentage of cells expressing green fluorescence as determined using flow cytometry represents the transfection efficiency. ${ }^{*} \mathrm{P}<0.01$, compared with the cells transfected with H_ANXA3-sh2.

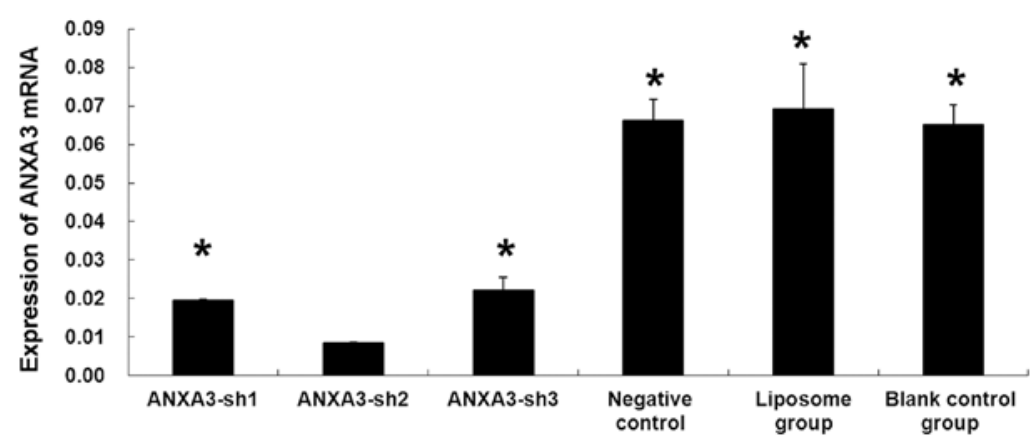

Figure 7. Assessment of the expression of ANXA 3 mRNA in the transfected cells using fluorescence quantitative RT-PCR; $\mathrm{P}<0.05$, compared with the expression of ANXA3 mRNA in the MDA-MB-231 cells transfected with H_ANXA3-sh2.

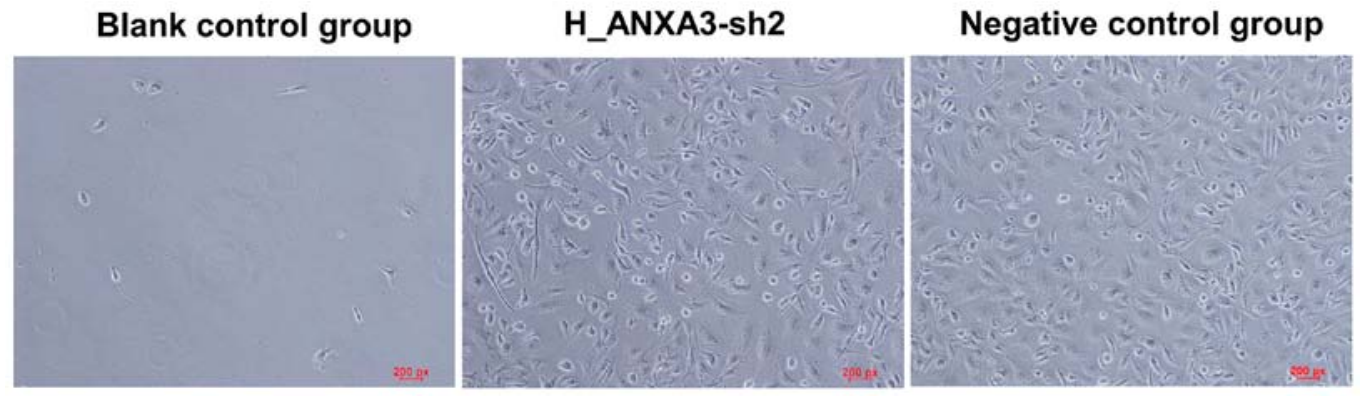

Figure 8. Cells treated with $600 \mathrm{ng} / \mathrm{ml}$ puromycin for 14 days (magnification, $\mathrm{x} 100$ ).

in the H_ANXA3-sh2 group was the highest, and the differences from all the other groups were statistically significant $(\mathrm{P}<0.01$; Fig. 6).

Assessing the expression of ANXA3 mRNA in the transfected cells using fluorescence quantitative RT-PCR. The results showed that the ANXA3 mRNA level in the MDA-MB-231 cells transfected with H_ANXA3-sh1, H_ANXA3-sh2, H_ANXA3-sh3, and negative control plasmid was $0.0196 \pm 0.0002,0.0085 \pm 0.0002,0.0220 \pm 0.0035$, and $0.0661 \pm 0.0057$, respectively, while the ANXA3 mRNA level in the blank control group and liposome group was $0.0692 \pm 0.0050$ and $0.0652 \pm 0.0118$, respectively. The expression of ANXA3 mRNA was significantly lower in the
MDA-MB-231 cells transfected with H_ANXA3-sh2 than in the other groups $(\mathrm{P}<0.05$; Fig. 7$)$, suggesting that the ANXA3 gene silencing effect of H_ANXA3-sh2 was the highest. Therefore, H_ANXA3-sh2 was chosen for the consequent experiments.

Identifying stably transfected cells using puromycin. After treatment with $600 \mathrm{ng} / \mathrm{ml}$ puromycin for 14 days, all cells in the blank control group died, while a large number of cells transfected with ANXA3-sh2 and negative control plasmids were viable (Fig. 8). The surviving cells were the stably transfected cells. The cells transfected with ANXA3-sh2 and negative control plasmids were named as MDA-MB-231-Sh and MDA-MB-231-NC cells, respectively. 

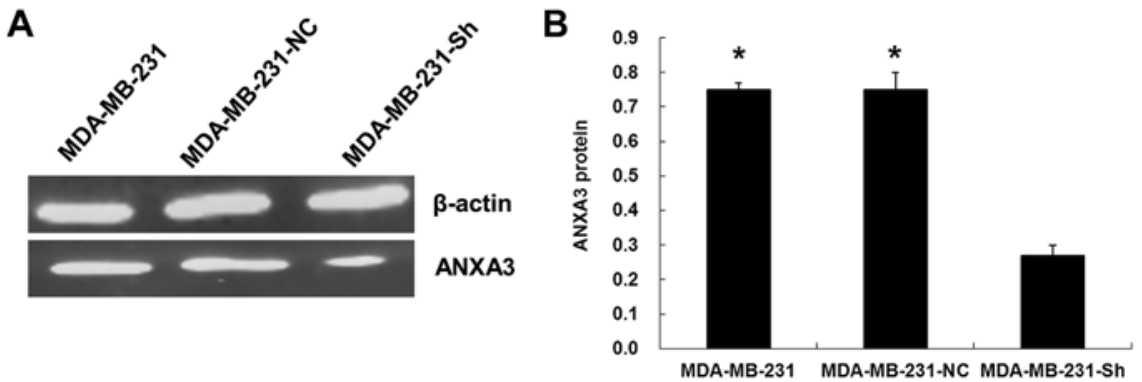

Figure 9. (A) Assessment of the expression of ANXA3 protein in the transfected cells using western blotting. (B) The expression of ANXA3 protein in the MDA-MB-231-Sh cells was significantly decreased, suggesting that the transfection of ANXA3-targeting shRNA significantly downregulated the expression of ANXA3 protein. " $\mathrm{P}<0.01$, compared with the MDA-MB-231-Sh cells.

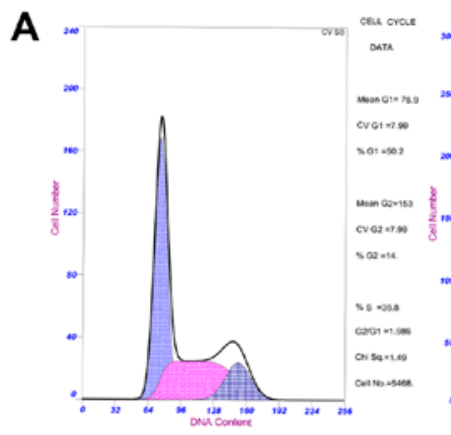

MDA-MB-231

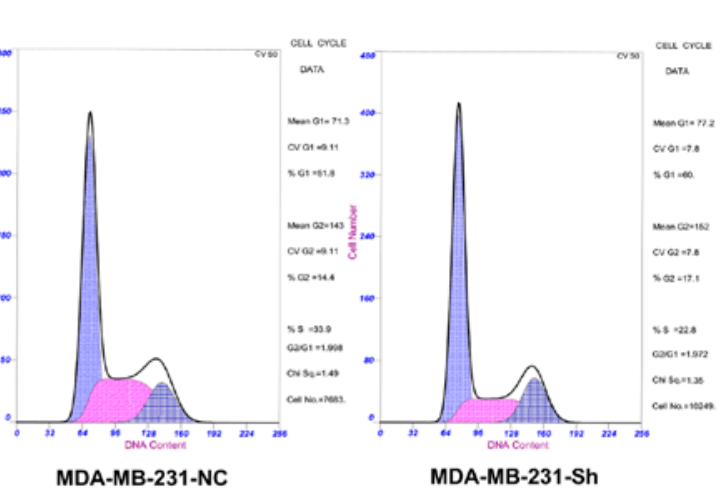

MDA-MB-231-NC
MDA-MB-231-Sh

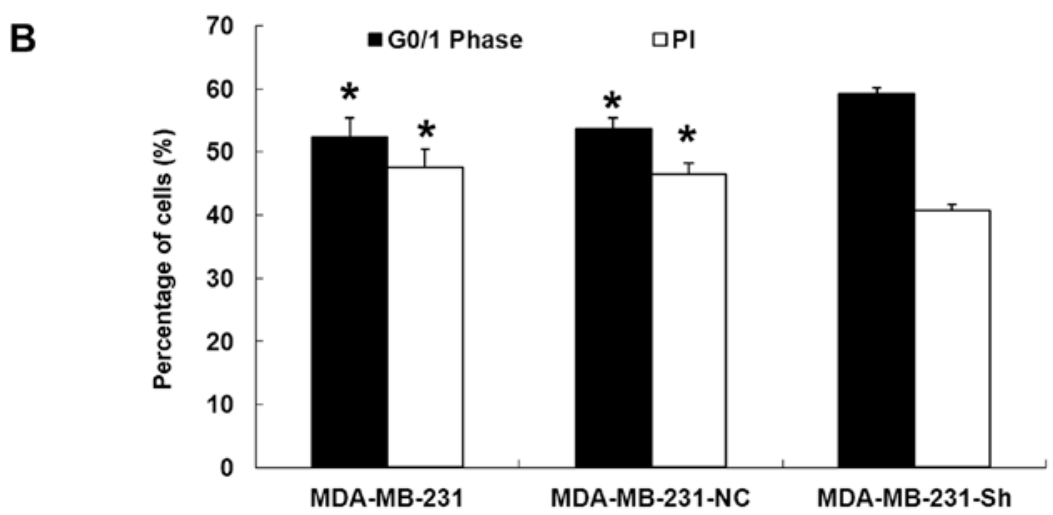

Figure 10. Evaluation of the cell cycle of the transfected cells using flow cytometry. (A) Assessment of the cell cycle in the transfected cells, negative control cells, and blank control cells. Propidium iodide staining was used to measure the DNA concentration. The Muticycle AV software was used to perform the fit analysis of the DNA cell cycle and calculate the percentage of cells in each phase. (B) The percentage of $\mathrm{G}_{0 / 1}$ cells in the MDA-MB-231-Sh cells was significantly higher, while PI was significantly lower, suggesting that the proliferation activity of the MDA-MB-231-Sh cells was significantly decreased. * $<0.05$, compared with the MDA-MB-231-Sh cells.

Assessing the expression of ANXA3 protein in the transfected cells using western blotting. The results showed that the expression of ANXA3 protein was significantly lower in the MDA-MB-231-Sh cells than that in the MDA-MB-231-NC and MDA-MB-231 cells $(\mathrm{P}<0.01$; Fig. 9).

Evaluating the cell cycle of the transfected cells using flow cytometry. The results showed that the percentage of cells in the $G_{0 / 1}$ phase was significantly higher in the MDA-MB-231-Sh group than in the MDA-MB-231-NC and MDA-MB-231 groups $(\mathrm{P}<0.05)$, while the proliferation index (PI; the percentage of the cells in the $S$ and $G_{2} / M$ phases among all the cells, which reflects the proliferation activities of the cells) was significantly lower in the MDA-MB-231-Sh cells than in the MDA-MB-231-NC and MDA-MB-231 cells $(P<0.05)$. However, the percentage of the cells in the $\mathrm{G}_{0 / 1}$ phase and PI in the MDA-MB-231-NC and MDA-MB-231 cells were not significantly different $(\mathrm{P}>0.05$; Fig. 10).

Evaluating the cell apoptosis rate using flow cytometry. The results showed that the cell apoptosis rate was significantly higher in the MDA-MB-231-Sh cells than that in the MDA-MB-231-NC and MDA-MB-231 cells $(\mathrm{P}<0.01)$. However, the apoptosis rate in the MDA-MB-231-NC and MDA-MB-231 cells was not significantly different $(\mathrm{P}>0.05$; Fig. 11). 
A
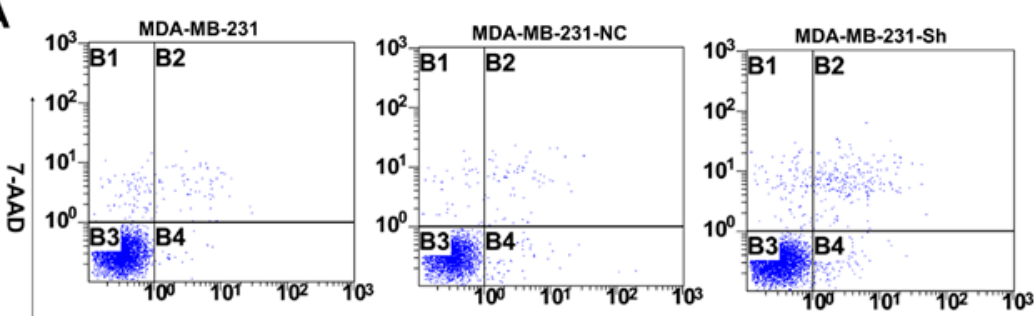

B

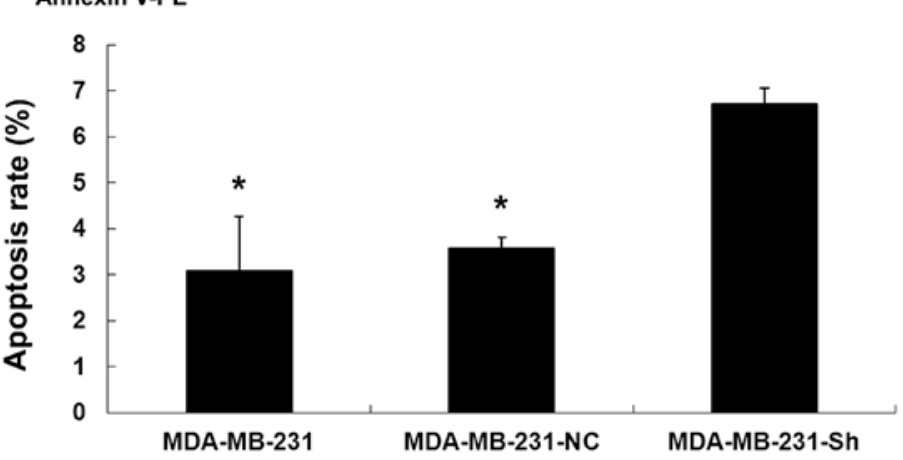

Figure 11. Evaluation of the cell apoptosis rate using flow cytometry. (A) Assessment of the apoptosis rate in the transfected cells, negative control cells, and blank control cells with flow cytometry. (B) The apoptosis rate in the MDA-MB-231-Sh cells was significantly increased. "P<0.05, compared with the MDAMB-231 and MDA-MB-231-NC cells.
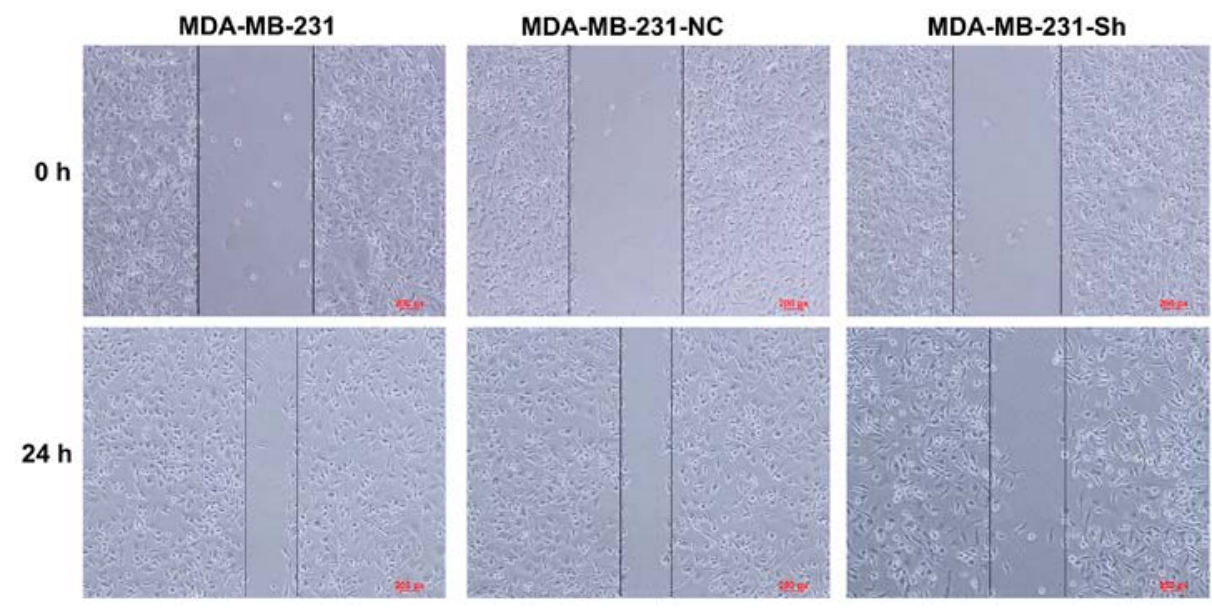

Figure 12. Evaluation of the cell migration ability using a wound healing assay by means of an inverted microscope (magnification, x100).
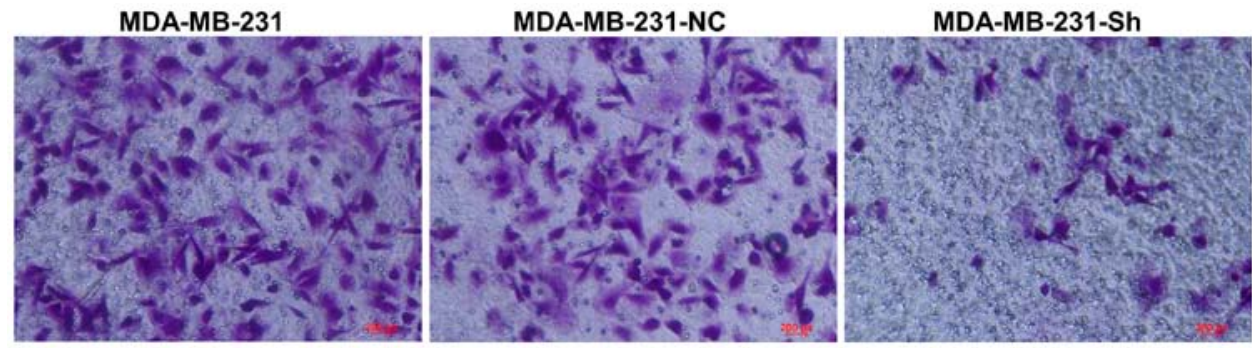

Figure 13. Evaluation of the cell invasion of the transfected cells using the Transwell assay by means of an inverted microscope (magnification, x200).

Evaluating cell migration using the wound healing assay. The wound healing assay showed that the migration ability of the MDA-MB-231-Sh cells was significantly lower than those of the MDA-MB-231-NC and MDA-MB-231 cells (Fig. 12).
Evaluating cell invasion using the Transwell assay. The Transwell assay showed that the number of MDA-MB-231-Sh cells invading the membrane was significantly lower than the number of invading MDA-MB-231-NC and MDA-MB-231 cells (Fig. 13). 


\section{Discussion}

Annexin A3 (ANXA3) is a member of the Annexin family. Previous studies have shown that the $A N X A 3$ gene is located at the 4q13-q22 of the human chromosome, and consists of 323 amino acid residues $(9,10)$. The change in expression of ANXA3 has important effects on the development, progression, drug resistance and metastasis of tumors. To date, only few studies have investigated the relationships between ANXA3 and malignant tumors, and the depth of these studies was not comparable with the investigations on other ANXA members such as ANXA1 and ANXA2. Liu et al (7) used fluorescence two-dimensional differential gel electrophoresis and liquid chromatography-mass spectrometry to screen the gastric cancer differentiation-associated protein, and found that $A N X A 3$ was closely related with the differentiation of gastric cancer cells. Xie et al (8) investigated the expression of ANXA3 in 60 patients with rectal cancer using immunohistochemical technology and found that the expression of ANXA3 protein in the rectal cancer tissues was upregulated. In addition, the overexpression of ANXA3 protein predicted the poor prognosis of the patients. Yip et al (6) used fluorescence quantitative PCR to compare the expression of seven biomarkers in the blood of North American and Malaysian patients with rectal cancers, and found that the level of ANXA3 in the blood was significantly higher in the patients with rectal cancer than the level in the healthy controls. Specifically, the level of ANXA3 in the blood was increased 2.06-fold in the Malaysian patients and 1.71-fold in the North American patients, both of which were statistically significant. These findings suggest that ANXA3 could be used as a biomarker for the diagnosis, treatment and outcome prediction for colorectal cancer. In a study performed by Lam et al (11), proteomic technologies were used to compare rectal cancer tissues and normal mucosa. The results showed that the expression of ANXA3 in the rectal cancer tissues was significantly upregulated. In a study in China, Zhu et al (12) reported that the RNA and protein levels of ANXA3 were higher in drug-resistant cell lines than in parental HT-29 cells. Zong et al (13) also found that the abnormal expression of ANXA3 was associated with the resistance of colorectal cancer to oxaliplatin, suggesting that ANXA3 could be used as a biomarker for drug-resistance. However, these studies were non-comprehensive, and hence further detailed studies are needed. Madoz-Gúrpide et al (14) found that the expression rate of ANXA3 in colorectal cancers was as high as $63 \%$, which was significantly higher than the rate in normal tissues. Baine et al (5) found that the expression of ANXA3 in pancreatic cancer tissues was 1.71fold of the level in healthy subjects. Liu et al (15) found that the expression of ANXA3 in lung cancer tissues with lymph node metastases was significantly higher than the expression in lung cancer tissues without lymph node metastases. Liang et al (16) reported that the expression of ANXA3 in the liver cancer cell line Hca-F with a high metastasis potential to lymph nodes was significantly upregulated, and the expression level was 2.3 -fold of the expression in the Hca-P cell line with a low metastasis potential to lymph nodes, suggesting that ANXA3 directly participates in the metastasis of liver cancer. Other studies $(17,18)$ also demonstrated that the expression of ANXA3 was positively associated with the axillary lymph node metastasis of tumors. These findings suggest that ANXA3 could play an important role in the development, proliferation, migration and metastasis of malignant tumors and/or tumor cells, which is in agreement with the present findings concerning the expression of ANXA3 in breast cancers. To date, few studies have investigated the expression of ANXA3 in breast cancers. The present study mainly investigated the effects of the expression of ANXA3 on the proliferation, migration and invasion of breast cancer MDA-MB-231 cells. The present study used fluorescence quantitative RT-PCR and western blotting to measure the expression of ANXA3 mRNA and protein in two breast cancer cell lines (MDAMB-231 and MCF-7). The results showed that the expression levels of ANXA3 mRNA and protein were significantly higher in the MDA-MB-231 cells than these levels in the MCF-7 cells, suggesting that the expression of ANXA3 in highly invasive breast cancer cells is increased. Three ANXA3 gene-targeting shRNA plasmids were successfully constructed and used to transfect the MDA-MB-231 cells for $48 \mathrm{~h}$ using the Lipofectamine transfection method. The results showed that the ANXA3 mRNA silencing effect of the ANXA3-sh2 plasmid was the highest (87.72\%). Then, puromycin was used to identify the stably transfected cells, in which the expression of ANXA3 protein was found to be significantly lower than the expression in the MDA-MB-231 cells using western blotting. The results of the flow cytometry showed that the percentage of the cells in the $G_{0 / 1}$ phase and the apoptosis rate were both significantly higher, while the PI was significantly lower in the MDA-MB-231-Sh cells than that in the MDA-MB-231-NC and MDA-MB-231 cells, suggesting that ANXA3 plays an important role in cell proliferation. The results of the wound healing assay showed that the migration ability of MDA-MB-231-Sh cells was significantly lower than that of the MDA-MB-231 cells. The Transwell assay also showed that fewer MDA-MB-231-Sh cells passed through the membrane compared with MDA-MB-231 cells, suggesting that ANXA3 also participated in the migration and metastasis of breast cancer cells, indicating that inhibiting the expression of ANXA3 significantly reduced the proliferation, migration, and invasion of breast cancer cells, and thus induced cell apoptosis and inhibited cell proliferation, migration and invasion. The findings of the present study were in agreement with the results reported by Zeng et al (17). The researchers used RNA interference technology to reduce the expression of ANXA3 in MCF-7 and MDA-MB-435 cells, and found that the proliferation of cancer cells was effectively inhibited and the apoptosis of the cells was evidently increased. In addition, the findings also suggested that the effects on cancer cell apoptosis could be associated with the effects of ANXA 3 on the regulation of the expression of BCL-2/Bax. In a study performed in China, Zhang et al (19) upregulated the expression of ANXA3 in gastric cancer MGC803 cells and found that upregulating the expression of ANXA3 not only enhanced the cell proliferation and colony formation ability, but also significantly inhibited the apoptosis of gastric cancer cells. These findings proved the participation of ANXA3 in regulating the proliferation and apoptosis of cancer cells, and thus in developing malignant tumors. However, the exact mechanisms underlying these effects still need to be investigated. 
The present study used RNA interference technology to silence the $A N X A 3$ gene in breast cancer MDA-MB-231 cells. It found that $A N X A 3$ could regulate the proliferation, apoptosis, invasion, and migration of breast cancer cells. Thus, this study provides the experimental basis for investigating the mechanisms involved in the development, progression, invasion and metastasis of breast cancer.

\section{References}

1. Bandorowicz-Pikuła J, Woś M and Pikuła S: Participation of annexins in signal transduction, regulation of plasma membrane structure and membrane repair mechanisms. Postepy Biochem 58: 135-148, 2012 (In Polish).

2. Fatimathas L and Moss SE: Annexins as disease modifiers. Histol Histopathol 25: 527-532, 2010.

3. Branishte T, Arsenescu-Georgescu C, Tomescu MC, Braniste A and Mitu F: Annexins, calcium-dependent phospholipid binding proteins in irreducible heart failure. Rev Med Chir Soc Med Nat Iasi 117: 648-653, 2013.

4. Bianchi C, Bombelli S, Raimondo F, Torsello B, Angeloni V, Ferrero S, Di Stefano V, Chinello C, Cifola I, Invernizzi L, et al: Primary cell cultures from human renal cortex and renal-cell carcinoma evidence a differential expression of two spliced isoforms of Annexin A3. Am J Pathol 176: 1660-1670, 2010.

5. Baine MJ, Chakraborty S, Smith LM, Mallya K, Sasson AR Brand RE and Batra SK: Transcriptional profiling of peripheral blood mononuclear cells in pancreatic cancer patients identifies novel genes with potential diagnostic utility. PLoS One 6: e17014, 2011.

6. Yip KT, Das PK, Suria D, Lim CR, Ng GH and Liew CC: A case-controlled validation study of a blood-based seven-gene biomarker panel for colorectal cancer in Malaysia. J Exp Clin Cancer Res 29: 128, 2010.

7. Liu Y, Li Y, Tan BB, Zhao Q, Fan LQ, Zhang ZD and Li ZX: Technique appraisement of comparative proteomics and screening of differentiation-related protein in gastric carcinoma. Hepatogastroenterology 60: 633-637, 2013.

8. Xie YQ, Fu D, He ZH and Tan QD: Prognostic value of Annexin A3 in human colorectal cancer and its correlation with hypoxia-inducible factor-1 $\alpha$. Oncol Lett 6: 1631-1635, 2013.
9. Wozny W, Schroer K, Schwall GP, Poznanović S, Stegmann W, Dietz K, Rogatsch H, Schaefer G, Huebl H, Klocker H, et al: Differential radioactive quantification of protein abundance ratios between benign and malignant prostate tissues: Cancer association of annexin A3. Proteomics 7: 313-322, 2007.

10. Yan XD, Pan LY, Yuan Y, Lang JH and Mao N: Identification of platinum-resistance associated proteins through proteomic analysis of human ovarian cancer cells and their platinum-resistant sublines. J Proteome Res 6: 772-780, 2007.

11. F Lam F, Jankova L, Dent OF, Molloy MP, Kwun SY, Clarke C, Chapuis P, Robertson G, Beale P, Clarke S, et al: Identification of distinctive protein expression patterns in colorectal adenoma. Proteomics Clin Appl 4: 60-70, 2010.

12. Zhu LQ, Shao JX, Sun JY, et al: Expression of ANXA3 in colorectal cancer cell lines. Modern Medical Journal 43: 267-271, 2015.

13. Zong MZ, Feng WT, Du N, Ye LL, Tao SD, Fu XH, He JD and Zhou JW: Screening and identifying oxaliplatin-resistanceassociated proteins in colorectal cancer cell lines. Tumor 33: 223-228, 2013.

14. Madoz-Gúrpide J, López-Serra P, Martínez-Torrecuadrada JL, Sánchez L, Lombardía L and Casal JI: Proteomics-based validation of genomic data: Applications in colorectal cancer diagnosis. Mol Cell Proteomics 5: 1471-1483, 2006.

15. Liu YF, Xiao ZQ, Li MX, Li MY, Zhang PF, Li C, Li F, Chen YH, Yi H, Yao HX, et al: Quantitative proteome analysis reveals annexin A3 as a novel biomarker in lung adenocarcinoma. J Pathol 217: 54-64, 2009.

16. Liang RC, Neo JC, Lo SL, Tan GS, Seow TK and Chung MC: Proteome database of hepatocellular carcinoma. J Chromatogr B Analyt Technol Biomed Life Sci 771: 303-328, 2002.

17. Zeng C, Ke Z, Song Y, Yao Y, Hu X, Zhang M, Li H and Yin J: Annexin A3 is associated with a poor prognosis in breast cancer and participates in the modulation of apoptosis in vitro by affecting the Bcl-2/Bax balance. Exp Mol Pathol 95: 23-31, 2013

18. Wu ZM, Qiu HJ and Fang SY: The expression of Annexin A3 in breast cancer and its significance. Zhong Liu Xue Za Zhi 20: 705-709, 2014

19. Zhang DY, Wang LD and Zeng C: Effects of high Annexin A3 expression on apoptosis and proliferation of gastric cancer MGC-803 cells. Lin Chuang Yu Shi Yan Bing Li Xue Za Zhi 31: 607-610, 2015 